\title{
DEATHS RECORDED AS MENTAL DISORDERS IN THE 15-24 AGE GROUP, 1979-89
}

$\mathrm{n}$ a recent Public Health Bulletin Supplement, Trends in

Major Causes of Death, NSW, 1971-1987, it was noted that Mental Disorders was recorded as one of the major causes of death among those in the 15-24 age group and had increased between 1979 and 1987. In 1987, 6 per cent of male and 7 per cent of female deaths in this age group were recorded under this classification. For both sexes it was the fourth most frequent cause of death, after motor vehicle crashes, unintentional injury and suicide for males, and after motor vehicle crashes, unintentional injury and other external causes, and of the same magnitude as suicide for females. Total deaths recorded as Mental Disorders rose from 24 in 1979 to 48 in 1987.

Mental Disorders appears to be a misleading label for a cause of death since such disorders cannot cause death but can only contribute to it. In view of its importance in youthful mortality, we decided to investigate how a death comes to be classified in this way, and which individual. causes most contribute to the classification.

\section{METHOD}

We used Australian Bureau of Statistics (ABS) death data as described in reference 1 . Cause of death is classified according to the International Classification of Diseases 9th Revision, and coded by the ABS. Since 1979 the classification Mental Disorders has come under the ICD-9 codes 2900-3199, which encompass psychotic conditions, neurotic, personality and other non-psychotic mental disorders and mental retardation. Alcohol dependence and drug dependence are classified under the subheading neurotic disorders, personality disorders and other nonpsychotic conditions: codes 3030-3039 and 3040-3049 respectively. The fourth digit refers to the specific substance in each case.

For a death to be coded under the alcohol or drug dependence rubric (and, hence, Mental Disorders), it must fulfil two criteria:

the primary cause of death must be alcohol or drug poisoning; and

it must be clear that such poisoning occurred as a result of a dependence on the substance.

The latter determination is necessarily subjective as it is based on what can be determined from the Coroner's notes. If there is not clearly a history of dependence, the death is coded as accidental. The relevant codes for poisoning by alcohol or drugs are 8500 (accidental poisoning by opiates and related narcotics), 8510-8519 (accidental poisoning by barbiturates), 8600 (accidental poisoning by alcoholic beverage) and 8609 (accidental poisoning by alcohol, unspecified). The latter are E-codes, which replace the injury and poisoning codes in cause of death coding.

We obtained the frequencies of each four-digit cause of death code for each of the years 1979 to 1987 , and two further years for which data has become available - 1988 and 1989 - for people in the 15-24 age group.

\section{RESULTS}

In all years most deaths recorded under the Mental Disorders classification were caused by poisoning by drugs, with a history of dependence (Table 1 ).

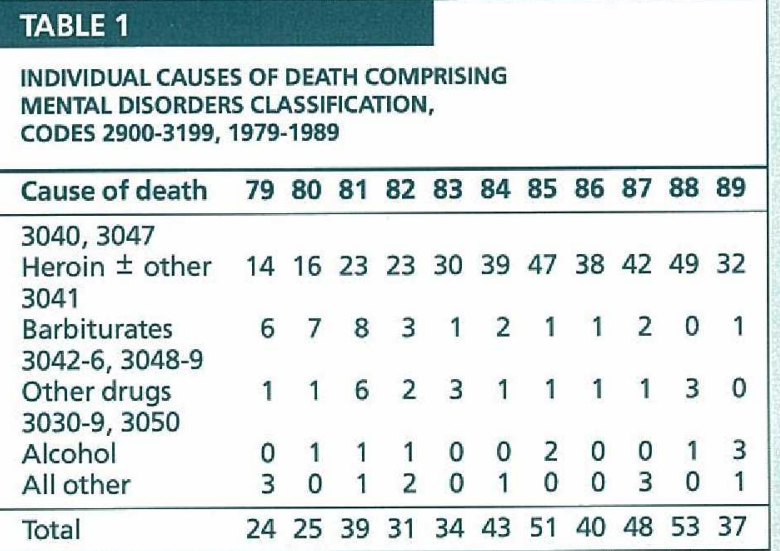

Code 3040 - dependence on morphine-type drugs comprising heroin, methadone, opium and derivatives, synthetics with morphine-like effects - predominated. Together with code 3047 (combinations of morphine-type drug with any other), it has been almost entirely responsible for the majority of deaths and the increase since 1979 in rates of death classified under Mental Disorders. Barbiturate dependence (code 3041) has declined, reflecting changes to the Poisons Act which restricted over-thecounter access to this drug

Over the 11-year period 385 deaths in the Mental Disorders classification were attributed to poisoning by drugs on which there was dependence. Of these 353 were morphinetype drugs and 32 barbiturates. The remainder of deaths classified under Mental Disorders totalled 19. Over the whole period nine of these deaths were attributed to alcohol dependence or abuse, four to anorexia nervosa, one coded as motor retardation and five as severe or profound mental retardation.

Examination of the accidental poisoning classifications for drugs and alcohol followed a similar pattern. Death due to accidental poisoning by morphine-type drugs has increased and by barbiturates decreased since 1979 . Over the whole period accidental poisoning by morphine-type drugs accounted for a further 77 deaths, by barbiturates a further 19 , and alcohol a further three deaths.

Suicide and self-inflicted poisoning by analgesics, antipyretics and antirheumatics (code 9500), in which category morphine-type drugs fall, accounted for 31 deaths over the period and has remained stable at about three a year, while suicide and self-inflicted poisoning by barbiturates (code 9501) fell from 17 in 1979 to eight in 1980 , and thereafter has remained at about three a year.

\section{Marilyn Rob}

Epidemiology and Health Services Evaluation Branch NSW Health Department 1. Fung SC, Lyle DM, Rob M. Trends in Major Causes of Death, NSW,
1971-1987. NSW Public Health Bulletin Supplement, Number 1, March $1971-$ 1992 